\title{
Autoconjugate representers for linear monotone operators
}

\author{
Heinz H. Bauschke; Xianfu Wang† and Liangjin Yaoł
}

February 10, 2008

\begin{abstract}
Monotone operators are of central importance in modern optimization and nonlinear analysis. Their study has been revolutionized lately, due to the systematic use of the Fitzpatrick function. Pioneered by Penot and Svaiter, a topic of recent interest has been the representation of maximal monotone operators by so-called autoconjugate functions. Two explicit constructions were proposed, the first by Penot and Zălinescu in 2005, and another by Bauschke and Wang in 2007. The former requires a mild constraint qualification while the latter is based on the proximal average.

We show that these two autoconjugate representers must coincide for continuous linear monotone operators on reflexive spaces. The continuity and the linearity assumption are both essential as examples of discontinuous linear operators and of subdifferential operators illustrate. Furthermore, we also construct an infinite family of autoconjugate representers for the identity operator on the real line.
\end{abstract}

2000 Mathematics Subject Classification: Primary 47H05; Secondary 47N10, 54A41.

Keywords: Autoconjugate representer, convex function, Fenchel conjugate, Fitzpatrick function, linear monotone operator, maximal monotone operator, subdifferential operator.

\section{Introduction}

Throughout this paper, we assume that

$X$ is a real reflexive Banach space, with continuous dual space $X^{*}$, and pairing $\langle\cdot, \cdot\rangle$.

\footnotetext{
*Mathematics, Irving K. Barber School, UBC Okanagan, Kelowna, British Columbia V1V 1V7, Canada. E-mail: heinz. bauschke@ubc.ca.

${ }^{\dagger}$ Mathematics, Irving K. Barber School, UBC Okanagan, Kelowna, British Columbia V1V 1V7, Canada. E-mail: shawn. wang@ubc. ca.

${ }^{\ddagger}$ Mathematics, Irving K. Barber School, UBC Okanagan, Kelowna, British Columbia V1V 1V7, Canada. E-mail: ljinyao@interchange.ubc.ca.
} 
The norm of $X$ is denoted by $\|\cdot\|$, and the norm in the dual space $X^{*}$ by $\|\cdot\|_{*}$.

Let $A: X \rightrightarrows X^{*}$ be a set-valued operator, with graph gra $A=\left\{\left(x, x^{*}\right) \in X \times X^{*} \mid x^{*} \in A x\right\}$, with inverse operator $A^{-1}: X^{*} \rightrightarrows X$ given by gra $A^{-1}=\left\{\left(x^{*}, x\right) \in X^{*} \times X \mid x^{*} \in A x\right\}$, with domain $\operatorname{dom} A=\{x \in X \mid A x \neq \varnothing\}$, and with range $\operatorname{ran} A=A(X)$. Recall that $A$ is monotone if

$$
\left(\forall\left(x, x^{*}\right) \in \operatorname{gra} A\right)\left(\forall\left(y, y^{*}\right) \in \operatorname{gra} A\right) \quad\left\langle x-y, x^{*}-y^{*}\right\rangle \geq 0 .
$$

A monotone operator $A$ is maximal monotone if no proper enlargement (in the sense of graph inclusion) of $A$ is monotone. Monotone operators are ubiquitous in Optimization and Analysis (see, e.g., [19, 31, 35, 36, 40, 45]) since they contain the key classes of subdifferential operators and of positive linear operators.

In [21], Fitzpatrick introduced the following tool in the study of monotone operators.

Definition 1.1 Let $A$ : $X \rightrightarrows X^{*}$. The Fitzpatrick function of $A$ is

$$
F_{A}:\left(x, x^{*}\right) \mapsto \sup _{\left(y, y^{*}\right) \in \operatorname{gra} A}\left\langle x, y^{*}\right\rangle+\left\langle y, x^{*}\right\rangle-\left\langle y, y^{*}\right\rangle
$$

Monotone Operator Theory has been revolutionized through the systematic use of the Fitzpatrick function; new results have been obtained and previously known result have been reproved in a simpler fashion - see, e.g., [1, 2, 3, 6, 8, 9, 10, 12, 13, 14, 15, 16, 17, 18, 22, 23, 26, 27, 28, 29, 30, 33, 37, 38, 39, 41, 42, 43, 44, 46]. Before listing some of the key properties of the Fitzpatrick function, we introduce a convenient notation utilized by Penot [29]: If $\left.\left.F: X \times X^{*} \rightarrow\right]-\infty,+\infty\right]$, set

$$
F^{\top}: X^{*} \times X:\left(x^{*}, x\right) \mapsto F\left(x, x^{*}\right),
$$

and similarly for a function defined on $X^{*} \times X$. We now define an associated operator $X \rightrightarrows X^{*}$ by requiring that for $\left(x, x^{*}\right) \in X \times X^{*}$,

$$
x^{*} \in G(F) x \quad \Leftrightarrow \quad F\left(x, x^{*}\right)=\left\langle x, x^{*}\right\rangle
$$

we also say that $F$ is a representer for $G(F)$.

Fact 1.2 (See [21].) Let $A: X \rightrightarrows X^{*}$ be maximal monotone. Then the following hold.

(i) $F_{A}$ is proper, lower semicontinuous, and convex.

(ii) $F_{A^{-1}}=F_{A}^{\top}$.

(iii) $F_{A} \geq\langle\cdot, \cdot\rangle$.

(iv) $A=G\left(F_{A}\right)$. 
Item (iv) of Fact 1.2 states the key property that the Fitzpatrick function $F_{A}$ is a representer for the maximal monotone operator $A$. It turns out that there are even more structured representers for $A$ available: recall that $\left.\left.F: X \times X^{*} \rightarrow\right]-\infty,+\infty\right]$ is autoconjugate, if

$$
F^{*}=F^{\top} .
$$

Autoconjugate representers are readily available for two important classes of maximal monotone operators.

Example 1.3 (subdifferential operator) Let $f: X \rightarrow]-\infty,+\infty]$ be proper, lower semicontinuous, and convex. Then the separable sum of $f$ and the Fenchel conjugate $f^{*}$, i.e.,

$$
\left.\left.f \oplus f^{*}: X \times X^{*} \rightarrow\right]-\infty,+\infty\right]:\left(x, x^{*}\right) \mapsto f(x)+f^{*}\left(x^{*}\right),
$$

is an autoconjugate representer for the subdifferential operator $\partial f$.

Example 1.4 (antisymmetric operator) Let $A: X \rightarrow X^{*}$ be continuous, linear, and antisymmetric, i.e., $A^{*}=-A$. Then the indicator function of the graph of $A$, i.e.,

$$
\left.\left.\iota_{\text {gra } A}: X \times X^{*} \rightarrow\right]-\infty,+\infty\right]:\left(x, x^{*}\right) \mapsto \begin{cases}0, & \text { if } x^{*}=A x \\ +\infty, & \text { otherwise }\end{cases}
$$

is an autoconjugate representer for $A$.

We now list some very pleasing and well known properties of autoconjugate functions.

Fact 1.5 (Penot-Simons-Zălinescu) (See [28, 29, 30, 42].) Let $\left.\left.F: X \times X^{*} \rightarrow\right]-\infty,+\infty\right]$ be autoconjugate. Then the following hold.

(i) $F$ is proper, lower semicontinuous, and convex.

(ii) $F \geq\langle\cdot, \cdot\rangle$.

(iii) $G(F)$ is maximal monotone.

(iv) If $\left.\left.\widetilde{F}: X \times X^{*} \rightarrow\right]-\infty,+\infty\right]$ is autoconjugate and $\widetilde{F} \leq F$, then $\widetilde{F}=F$.

Unfortunately, the Fitzpatrick function $F_{A}$ is usually not an autoconjugate representer for $A$. In view of Fact 1.5)(iii), it was tempting to ask whether every general maximal monotone operator possesses an autoconjugate representer. Nonconstructive existence proofs were presented by Penot [28, 29] and by Svaiter [43] in 2003 (see also Ghoussoub's preprint [23]). The first actual construction of an autoconjugate representer for a maximal monotone operator satisfying a mild constraint qualification was provided by Penot and Zălinescu in 2005. 
Fact 1.6 (Penot-Zălinescu) (See [30].) Let $A: X \rightrightarrows X^{*}$ be maximal monotone. Suppose that the affine hull of $\operatorname{dom} A$ is closed. Then

$$
\begin{aligned}
\mathcal{A}_{A}: X \times X^{*} & \rightarrow]-\infty,+\infty] \\
\left(x, x^{*}\right) & \mapsto \inf _{y^{*} \in X^{*}} \frac{1}{2} F_{A}\left(x, x^{*}+y^{*}\right)+\frac{1}{2} F_{A}^{* \top}\left(x, x^{*}-y^{*}\right)
\end{aligned}
$$

is an autoconjugate representer for $A$.

Another autoconjugate representer was very recently proposed in [9]. While this proximalaveraged based construction is more involved [4, 5, 7], it has the advantage of not imposing a constraint qualification.

Fact 1.7 (See 9 .) Let $A: X \rightrightarrows X^{*}$ be maximal monotone. Then

$$
\begin{aligned}
\mathcal{B}_{A}: X \times X^{*} & \rightarrow]-\infty,+\infty] \\
\left(x, x^{*}\right) & \mapsto \inf _{\left(y, y^{*}\right) \in X \times X^{*}} \frac{1}{2} F_{A}\left(x+y, x^{*}+y^{*}\right)+\frac{1}{2} F_{A}^{* \top}\left(x-y, x^{*}-y^{*}\right)+\frac{1}{2}\|y\|^{2}+\frac{1}{2}\left\|y^{*}\right\|_{*}^{2}
\end{aligned}
$$

is an autoconjugate representer for $A$.

It is natural to ask "How do the autoconjugate representers $\mathcal{A}_{A}$ and $\mathcal{B}_{A}$ compare?" We provide two answers to this question: First, we show that if $A: X \rightarrow X^{*}$ is continuous, linear, and monotone, then $\mathcal{A}_{A}$ and $\mathcal{B}_{A}$ coincide; furthermore, we provide a formula for this autoconjugate representer which agrees with a third autoconjugate representer $\mathcal{C}_{A}$ that is contained in the work by Ghoussoub (Theorem 3.1). Secondly, for nonlinear monotone subdifferential operators, the two autoconjugate representers may be different (Theorem 5.1).

The first answer raises the question on whether autoconjugate representers for continuous linear monotone operators are unique. We answer this question in the negative by providing a family of autoconjugate representers for the identity operator Id (Theorem 4.2). However, we show that the autoconjugate representers $\mathcal{A}_{A}$ and $\mathcal{B}_{A}$ in this setting are characterized by a pleasing symmetry property (Theorem 4.4).

We conclude by discussing discontinuous linear monotone operators. It turns out that $\mathcal{A}_{A}$ may fail to be autoconjugate (Example 6.5), which underlines not only the continuity assumption in Theorem 3.1 but also the importance of the constraint qualification in Fact 1.6 .

The remainder of this paper is organized as follows. Section 2 contains some results on quadratic functions and another autoconjugate representer that will be used in later sections. In Section 3 , we show that $\mathcal{A}_{A}$ and $\mathcal{B}_{A}$ coincide and provide a simple formula for it. Uniqueness of autoconjugate representations are discussed in Section 4, and a characterization in the symmetric case is also presented. In stark contrast, and as shown in Section $5, \mathcal{A}_{A}$ and $\mathcal{B}_{A}$ may be different for (nonlinear) subdifferential operators. The final Section 6 reveals similar difference for discontinuous linear operators. 
Notation utilized is standard as in Convex Analysis and Monotone Operator Theory; see, e.g., [34, 35, 45. Thus, for a proper convex function $f: X \rightarrow]-\infty,+\infty]$, we write $f^{*}: x^{*} \mapsto \sup _{x \in X}\left\langle x, x^{*}\right\rangle-$ $f(x), \partial f: X \rightrightarrows X^{*}: x \mapsto\left\{x^{*} \in X^{*} \mid(\forall y \in X)\left\langle y-x, x^{*}\right\rangle+f(x) \leq f(y)\right\}$, and $\operatorname{dom} f=\{x \in X \mid$ $f(x)<+\infty\}$, for the Fenchel conjugate, subdifferential operator, and domain of $f$, respectively. The strictly positive integers are $\mathbb{N}=\{1,2, \ldots\}$.

\section{Auxiliary Results}

The following result in a consequence of results and proof techniques introduced by Penot, Simons, and Zălinescu [30, 42]. It also extends [23, Lemma 2.2].

Proposition 2.1 Let $F_{1}$ and $F_{2}$ be autoconjugate functions on $X \times X^{*}$ representing maximal monotone operators $A_{1}$ and $A_{2}$, respectively. Suppose that

$$
\bigcup_{\lambda>0} \lambda\left(P_{X} \operatorname{dom} F_{1}-P_{X} \operatorname{dom} F_{2}\right) \quad \text { is a closed subspace of } X
$$

where $P_{X}: X \times X^{*} \rightarrow X:\left(x, x^{*}\right) \mapsto x$, and set

$$
\left.\left.F: X \times X^{*} \rightarrow\right]-\infty,+\infty\right]:\left(x, x^{*}\right) \mapsto \inf _{y^{*} \in X^{*}} F_{1}\left(x, y^{*}\right)+F_{2}\left(x, x^{*}-y^{*}\right) .
$$

Then $F$ is an autoconjugate representer for $A_{1}+A_{2}$, and the infimum in (11) is attained.

Proof. Let $\left(x, x^{*}\right) \in X \times X^{*}$. Using Simons and Zălinescu's [42, Theorem 4.2] and the assumption that each $F_{i}$ is autoconjugate, we obtain

$$
\begin{aligned}
F^{*}\left(x^{*}, x\right) & =\min _{x_{1}^{*}+x_{2}^{*}=x^{*}} F_{1}^{*}\left(x_{1}^{*}, x\right)+F_{2}^{*}\left(x_{2}^{*}, x\right) \\
& =\min _{x_{1}^{*}+x_{2}^{*}=x^{*}} F_{1}\left(x, x_{1}^{*}\right)+F_{2}\left(x, x_{2}^{*}\right) \\
& =F\left(x, x^{*}\right) .
\end{aligned}
$$

Thus, $F$ is autoconjugate and the infimum in (11) is attained.

It remains to show that $G(F)=G\left(F_{1}\right)+G\left(F_{2}\right)$. Since autoconjugates are greater than or equal to $\langle\cdot, \cdot\rangle$ (see Fact 1.5](ii)], the above implies the equivalences

$$
\begin{aligned}
x^{*} \in G(F) x & \Leftrightarrow F\left(x, x^{*}\right)=\left\langle x, x^{*}\right\rangle \\
& \Leftrightarrow\left(\exists y^{*} \in X^{*}\right) \quad F_{1}\left(x, y^{*}\right)+F_{2}\left(x, x^{*}-y^{*}\right)=\left\langle x, y^{*}\right\rangle+\left\langle x, x^{*}-y^{*}\right\rangle \\
& \Leftrightarrow\left(\exists y^{*} \in X^{*}\right) \quad F_{1}\left(x, y^{*}\right)=\left\langle x, y^{*}\right\rangle \text { and } F_{2}\left(x, x^{*}-y^{*}\right)=\left\langle x, x^{*}-y^{*}\right\rangle \\
& \Leftrightarrow\left(\exists y^{*} \in X^{*}\right) y^{*} \in G\left(F_{1}\right)(x) \text { and } x^{*}-y^{*} \in G\left(F_{2}\right)(x) \\
& \Leftrightarrow\left(\exists y^{*} \in X^{*}\right) y^{*} \in A_{1} x \text { and } x^{*}-y^{*} \in A_{2} x \\
& \Leftrightarrow x^{*} \in\left(A_{1}+A_{2}\right) x .
\end{aligned}
$$


Therefore, $G(F)=A_{1}+A_{2}$, i.e., $F$ is a representer for $A_{1}+A_{2}$.

Suppose that

$$
A: X \rightarrow X^{*} \text { is linear and continuous. }
$$

Then $A$ is symmetric (resp. antisymmetric) if $A^{*}=A$ (resp. $A^{*}=-A$ ). We denote the symmetric part and the antisymmetric part of $A$ by

$$
A_{+}=\frac{1}{2} A+\frac{1}{2} A^{*} \quad \text { and } \quad A_{\circ}=\frac{1}{2} A-\frac{1}{2} A^{*},
$$

respectively. Throughout, we shall work with the quadratic function

$$
q_{A}: X \rightarrow \mathbb{R}: x \mapsto \frac{1}{2}\langle x, A x\rangle,
$$

and we will use the well known facts (see, e.g., [32]) that $q_{A}=q_{A_{+}}$, that

$$
\nabla q_{A}=A_{+},
$$

and that $A$ is maximal monotone if and only if $q_{A}$ is convex. The next result provides a formula for $q_{A}^{*}$ that will be useful later.

Proposition 2.2 Let $A: X \rightarrow X^{*}$ be continuous, linear, symmetric, and monotone. Then

$$
\left(\forall\left(x, x^{*}\right) \in X \times X^{*}\right) \quad q_{A}^{*}\left(x^{*}+A x\right)=q_{A}(x)+\left\langle x, x^{*}\right\rangle+q_{A}^{*}\left(x^{*}\right)
$$

and

$$
q_{A}^{*} \circ A=q_{A}
$$

Proof. Let $\left(x, x^{*}\right) \in X \times X^{*}$. Then

$$
\begin{aligned}
q_{A}^{*}\left(x^{*}+A x\right) & =\sup _{y}\left\langle y, x^{*}+A x\right\rangle-q_{A}(y)=\sup _{y}\left\langle y, x^{*}\right\rangle-q_{A}(y)+\langle y, A x\rangle \\
& =q_{A}(x)+\sup _{y}\left\langle y, x^{*}\right\rangle-q_{A}(y)+\langle y, A x\rangle-q_{A}(x)=q_{A}(x)+\sup _{y}\left\langle y, x^{*}\right\rangle-q_{A}(y-x) \\
& =q_{A}(x)+\left\langle x, x^{*}\right\rangle+\sup _{y}\left\langle y-x, x^{*}\right\rangle-q_{A}(y-x)=q_{A}(x)+\left\langle x, x^{*}\right\rangle+q_{A}^{*}\left(x^{*}\right),
\end{aligned}
$$

which verifies (18). To see (19), set $x^{*}=0$ in (18).

Proposition 2.3 Let $A: X \rightarrow X^{*}$ be continuous, linear, and monotone, and let $\left(x, x^{*}\right) \in X \times X^{*}$. Then

$$
F_{A}\left(x, x^{*}\right)=2 q_{A_{+}}^{*}\left(\frac{1}{2} x^{*}+\frac{1}{2} A^{*} x\right)=\frac{1}{2} q_{A_{+}}^{*}\left(x^{*}+A^{*} x\right) .
$$

and

$$
F_{A}^{*}\left(x^{*}, x\right)=\iota_{\text {gra } A}\left(x, x^{*}\right)+\langle x, A x\rangle .
$$


Proof. As in the proof of [3, Theorem 2.3(i)], we have

$$
\begin{aligned}
F_{A}\left(x, x^{*}\right) & =\sup _{y \in X}\langle x, A y\rangle+\left\langle y, x^{*}\right\rangle-\langle y, A y\rangle \\
& =2 \sup _{y \in X}\left\langle y, \frac{1}{2} x^{*}+\frac{1}{2} A^{*} x\right\rangle-q_{A_{+}}(y) \\
& =2 q_{A_{+}}^{*}\left(\frac{1}{2} x^{*}+\frac{1}{2} A^{*} x\right) \\
& =\frac{1}{2} q_{A_{+}}^{*}\left(x^{*}+A^{*} x\right) .
\end{aligned}
$$

This verifies (21). Furthermore, (22) follows from $F_{A}^{*}\left(x^{*}, x\right)=\left(\iota_{\operatorname{gra} A}+\langle\cdot, \cdot\rangle\right)^{*} \mathrm{~T}^{*}\left(x^{*}, x\right)=$ $\iota_{\text {gra } A}\left(x, x^{*}\right)+\langle x, A x\rangle$.

Proposition 2.4 Let $\left.\left.F_{1}: X \times X^{*} \rightarrow\right]-\infty,+\infty\right]$ be autoconjugate, and let $A_{2}: X \rightarrow X^{*}$ be continuous, linear, and antisymmetric. Then the function

$$
\left(x, x^{*}\right) \mapsto F_{1}\left(x, x^{*}-A_{2} x\right)
$$

is an autoconjugate representer for $G\left(F_{1}\right)+A_{2}$.

Proof. Set $F_{2}=\iota_{\text {gra } A_{2}}$. By Example 1.4, $F_{2}$ is an autoconjugate representer for $A_{2}$. Let $F$ be as in Proposition 2.1. Then for every $\left(x, x^{*}\right) \in X \times X^{*}$, we have

$$
\begin{aligned}
F\left(x, x^{*}\right) & =\inf _{z^{*} \in X^{*}} F_{1}\left(x, x^{*}-z^{*}\right)+F_{2}\left(x, z^{*}\right) \\
& =\inf _{z^{*} \in X^{*}} F_{1}\left(x, x^{*}-z^{*}\right)+\iota_{\text {gra }} A_{2}\left(x, z^{*}\right) \\
& =F_{1}\left(x, x^{*}-A_{2} x\right) .
\end{aligned}
$$

Thus, Proposition 2.1 yields that $F$ represents $G\left(F_{1}\right)+A_{2}$.

Example 2.5 (Ghoussoub) (See also [23, Section 1].) Let $f: X \rightarrow]-\infty,+\infty]$ be proper, lower semicontinuous, and convex, and let $A$ be antisymmetric. Then the function

$$
\left(x, x^{*}\right) \mapsto f(x)+f^{*}\left(x^{*}-A x\right)
$$

is an autoconjugate representer for $\partial f+A$.

Proof. By Example 1.3, $f \oplus f^{*}$ is an autoconjugate representer for $\partial f$. The result thus follows from Proposition 2.4.

Corollary 2.6 Let $A: X \rightarrow X^{*}$ be continuous, linear, and monotone. Then

$$
\begin{aligned}
\mathcal{C}_{A}: X \times X^{*} & \rightarrow]-\infty,+\infty] \\
\left(x, x^{*}\right) & \mapsto q_{A_{+}}(x)+q_{A_{+}}^{*}\left(x^{*}-A_{\circ} x\right)
\end{aligned}
$$

is an autoconjugate representer for $A$. In particular, if $A$ is symmetric, then

$$
\mathcal{C}_{A}=q_{A} \oplus q_{A}^{*}
$$


Proof. This follows from (17) and Example 2.5 (when applied to the function $q_{A}=q_{A_{+}}$and to the antisymmetric operator $\left.A_{\circ}\right)$.

We now show that the Ghoussoub representers are closed under the partial infimal convolution operation of Proposition 2.1.

Proposition 2.7 Let $A$ and $B$ be continuous, linear, and monotone on $X$. Then the function

$$
\left.\left.F: X \times X^{*} \rightarrow\right]-\infty,+\infty\right]:\left(x, x^{*}\right) \mapsto \min _{y^{*} \in X^{*}} \mathcal{C}_{A}\left(x, x^{*}-y^{*}\right)+\mathcal{C}_{B}\left(x, y^{*}\right)
$$

coincides with the autoconjugate representer $\mathcal{C}_{A+B}$ for $A+B$.

Proof. In view of Proposition 2.1, we only need to show that $F=\mathcal{C}_{A+B}$. Let $\left(x, x^{*}\right) \in X \times X^{*}$. Using (29) and Corollary 2.6, we obtain

$$
\begin{aligned}
F\left(x, x^{*}\right) & =\min _{y^{*} \in X^{*}} q_{A_{+}}(x)+q_{A_{+}}^{*}\left(x^{*}-y^{*}-A_{\circ} x\right)+q_{B_{+}}(x)+q_{B_{+}}^{*}\left(y^{*}-B_{\circ} x\right) \\
& =q_{A_{+}}(x)+q_{B_{+}}(x)+\left(q_{A_{+}}^{*} \square q_{B_{+}}^{*}\right)\left(x^{*}-A_{\circ} x-B_{\circ} x\right) \\
& =q_{A_{+}+B_{+}}(x)+\left(q_{A_{+}}+q_{B_{+}}\right)^{*}\left(x^{*}-A_{\circ} x-B_{\circ} x\right) \\
& =q_{(A+B)_{+}}(x)+q_{(A+B)_{+}}^{*}\left(x^{*}-(A+B)_{\circ} x\right) \\
& =\mathcal{C}_{A+B}\left(x, x^{*}\right)
\end{aligned}
$$

as required.

\section{Coincidence}

We are now ready for one of our main results.

Theorem 3.1 (coincidence) Let $A: X \rightarrow X^{*}$ be continuous, linear, and monotone. Then all three autoconjugate representers $\mathcal{A}_{A}, \mathcal{B}_{A}, \mathcal{C}_{A}$ for $A$ coincide with the function

$$
\left(x, x^{*}\right) \mapsto\left\langle x, x^{*}\right\rangle+q_{A_{+}}^{*}\left(x^{*}-A x\right) .
$$

Proof. The proof proceeds by proving a succession of claims. Let $\left(x, x^{*}\right) \in X \times X^{*}$. 
Claim 1: $\mathcal{A}_{A}=\mathcal{C}_{A}$.

Using (8), (22), and (27), we obtain

$$
\begin{aligned}
\mathcal{A}_{A}\left(x, x^{*}\right) & \left.=\inf _{y^{*} \in X^{*}} \frac{1}{2} F_{A}\left(x, x^{*}+y^{*}\right)\right)+\frac{1}{2} F_{A}^{*}\left(x^{*}-y^{*}, x\right) \\
& \left.=\inf _{y^{*} \in X^{*}} \frac{1}{2} F_{A}\left(x, x^{*}+y^{*}\right)\right)+\iota_{\mathrm{gra}}\left(x, x^{*}-y^{*}\right)+q_{A}(x) \\
& =\frac{1}{2} F_{A}\left(x, 2 x^{*}-A x\right)+q_{A_{+}}(x) \\
& =q_{A_{+}}^{*}\left(x^{*}-\frac{1}{2} A x+\frac{1}{2} A^{*} x\right)+q_{A_{+}}(x) \\
& =q_{A_{+}}^{*}\left(x^{*}-A_{\circ} x\right)+q_{A_{+}}(x) \\
& =\mathcal{C}_{A}\left(x, x^{*}\right) .
\end{aligned}
$$

This verifies Claim 1.

Claim 2: $\mathcal{A}_{A}$ coincides with the function of (31).

In view of (32) and Proposition 2.2, we see that

$$
\begin{aligned}
\mathcal{A}_{A}\left(x, x^{*}\right) & =q_{A_{+}}^{*}\left(x^{*}-\frac{1}{2} A x+\frac{1}{2} A^{*} x\right)+q_{A_{+}}(x) \\
& =q_{A_{+}}^{*}\left(x^{*}-A x+A_{+} x\right)+q_{A_{+}}(x) \\
& =2 q_{A_{+}}(x)+\left\langle x, x^{*}-A x\right\rangle+q_{A_{+}}^{*}\left(x^{*}-A x\right) \\
& =\left\langle x, x^{*}\right\rangle+q_{A_{+}}^{*}\left(x^{*}-A x\right),
\end{aligned}
$$

which establishes Claim 2.

Claim 3: $\mathcal{A}_{A}=\mathcal{B}_{A}$.

Using (9), (22), (21), Proposition 2.2, and Claim 2, we have

$$
\begin{aligned}
& \mathcal{B}_{A}\left(x, x^{*}\right)= \inf _{\left(y, y^{*}\right) \in X \times X^{*}} \frac{1}{2} F_{A}\left(x+y, x^{*}+y^{*}\right)+\frac{1}{2} F_{A}^{* \top}\left(x-y, x^{*}-y^{*}\right)+\frac{1}{2}\|y\|^{2}+\frac{1}{2}\left\|y^{*}\right\|_{*}^{2} \\
&= \inf _{\left(y, y^{*}\right) \in X \times X^{*}} \frac{1}{2} F_{A}\left(x+y, x^{*}+y^{*}\right)+\iota_{\mathrm{gra} A}\left(x-y, x^{*}-y^{*}\right) \\
& \quad \quad \quad+\frac{1}{2}\langle x-y, A(x-y)\rangle+\frac{1}{2}\|y\|^{2}+\frac{1}{2}\left\|y^{*}\right\|_{*}^{2} \\
&=\inf _{y \in X} \frac{1}{2} F_{A}\left(x+y, 2 x^{*}-A(x-y)\right)+q_{A}(x-y)+\frac{1}{2}\|y\|^{2}+\frac{1}{2}\left\|x^{*}-A(x-y)\right\|_{*}^{2} \\
&=\inf _{y \in X} q_{A_{+}}^{*}\left(x^{*}-\frac{1}{2} A(x-y)+\frac{1}{2} A^{*}(x+y)\right)+q_{A_{+}}(x-y)+\frac{1}{2}\|y\|^{2}+\frac{1}{2}\left\|x^{*}-A(x-y)\right\|_{*}^{2} \\
&=\inf _{y \in X} q_{A_{+}}^{*}\left(x^{*}-A x+A_{+}(x+y)\right)+q_{A_{+}}(x-y)+\frac{1}{2}\|y\|^{2}+\frac{1}{2}\left\|x^{*}-A(x-y)\right\|_{*}^{2} \\
&=\inf _{y \in X} q_{A_{+}}^{*}\left(x^{*}-A x\right)+\left\langle x+y, x^{*}-A x\right\rangle+q_{A_{+}}(x+y)+q_{A_{+}}(x-y) \\
& \quad \quad \quad \quad \frac{1}{2}\|y\|^{2}+\frac{1}{2}\left\|x^{*}-A(x-y)\right\|_{*}^{2} \\
&=\inf _{y \in X} q_{A_{+}}^{*}\left(x^{*}-A x\right)+\left\langle x+y, x^{*}-A x\right\rangle+2 q_{A_{+}}(x)+2 q_{A_{+}}(y) \\
& \quad \quad \quad \quad \frac{1}{2}\|-y\|^{2}+\frac{1}{2}\left\|x^{*}-A(x-y)\right\|_{*}^{2} \\
& \geq q_{A_{+}}^{*}\left(x^{*}-A x\right)+\left\langle x, x^{*}\right\rangle+\inf _{y \in X}\left\langle y, x^{*}-A x\right\rangle+2 q_{A_{+}}(y)+\left\langle-y, x^{*}-A(x-y)\right\rangle
\end{aligned}
$$




$$
\begin{aligned}
& =q_{A_{+}}^{*}\left(x^{*}-A x\right)+\left\langle x, x^{*}\right\rangle+\inf _{y \in X} 2 q_{A_{+}}(y)+\langle-y, A y\rangle \\
& =q_{A_{+}}^{*}\left(x^{*}-A x\right)+\left\langle x, x^{*}\right\rangle \\
& =\mathcal{A}_{A}\left(x, x^{*}\right) .
\end{aligned}
$$

Hence $\mathcal{B}_{A} \geq \mathcal{A}_{A}$. On the other hand, both $\mathcal{A}_{A}$ and $\mathcal{B}_{A}$ are autoconjugate (see Fact 1.6 and Fact 1.7). Altogether, Fact 1.5](iv) implies Claim 3.

Finally, observe that Claims $1-3$ yield the result.

Example 3.2 Suppose that $X$ is the Euclidean plane $\mathbb{R}^{2}$, let $\theta \in\left[0, \frac{\pi}{2}[\right.$, and set

$$
A=\left(\begin{array}{cc}
\cos \theta & -\sin \theta \\
\sin \theta & \cos \theta
\end{array}\right) \text { and } A_{\pi / 2}=\left(\begin{array}{cc}
0 & -1 \\
1 & 0
\end{array}\right) .
$$

Then for every $\left(x, x^{*}\right) \in \mathbb{R}^{2} \times \mathbb{R}^{2}$,

$$
\begin{aligned}
\mathcal{A}_{A}\left(x, x^{*}\right) & =\mathcal{B}_{A}\left(x, x^{*}\right)=\mathcal{C}_{A}\left(x, x^{*}\right) \\
& =\frac{1}{2 \cos \theta}\left\|x^{*}-A x\right\|^{2}+\left\langle x, x^{*}\right\rangle \\
& =\frac{1}{2 \cos \theta}\left\|x^{*}-(\sin \theta) A_{\pi / 2} x\right\|^{2}+\frac{\cos \theta}{2}\|x\|^{2} .
\end{aligned}
$$

Proof. This follows from Theorem 3.1 since $A_{+}=(\cos \theta) \operatorname{Id}, q_{A_{+}}=(\cos \theta) \frac{1}{2}\|\cdot\|^{2}$, and $A_{\circ}=$ $(\sin \theta) A_{\pi / 2}$.

\section{Observations on Nonuniqueness}

Theorem 3.1 might nurture the conjecture that for continuous linear monotone operators, all autoconjugate representers coincide. This conjecture is false - we shall provide a whole family of distinct autoconjugate representers for the identity on $\mathbb{R}$. Our constructions rests on the following result.

Proposition 4.1 Let $g: \mathbb{R} \rightarrow]-\infty,+\infty]$ be such that

$$
(\forall x \in \mathbb{R}) \quad g^{*}(-x)=g(x) \geq 0 .
$$

Then

$$
g(0)=0 .
$$

Moreover, each of the following functions satisfies (38):

(i) the indicator function $\iota_{[0,+\infty[}: x \mapsto \begin{cases}0, & \text { if } x \geq 0 ; \\ +\infty, & \text { if } x<0 ;\end{cases}$ 
(ii) the energy function $\frac{1}{2}|\cdot|^{2}$;

(iii) for $p>1$ and $q>1$ such that $\frac{1}{p}+\frac{1}{q}=1$, the function $x \mapsto \begin{cases}\frac{1}{p} x^{p}, & \text { if } x \geq 0 \\ \frac{1}{q}(-x)^{q}, & \text { if } x<0\end{cases}$

Proof. On the one hand, $g(0) \geq 0$. On the other hand, $g(0)=g^{*}(-0)=g^{*}(0)=\sup _{y \in \mathbb{R}}-g(y)=$ $-\inf _{y \in \mathbb{R}} g(y) \leq 0$. Altogether, $g(0)=0$ and so (39). It is straight-forward to verify that each of the given functions satisfies (38).

Theorem 4.2 Let $g: \mathbb{R} \rightarrow]-\infty,+\infty]$ be such that for every $x \in \mathbb{R}, g^{*}(-x)=g(x) \geq 0$, and set $q: \mathbb{R} \rightarrow \mathbb{R}: x \mapsto \frac{1}{2}|x|^{2}$. Then

$$
\left.\left.F: \mathbb{R}^{2} \rightarrow\right]-\infty,+\infty\right]:(x, y) \mapsto q\left(\frac{x+y}{\sqrt{2}}\right)+g\left(\frac{x-y}{\sqrt{2}}\right)
$$

is an autoconjugate representer for $\mathrm{Id}: \mathbb{R} \rightarrow \mathbb{R}: x \mapsto x$.

Proof. Let $(x, y) \in \mathbb{R}^{2}$. Using the fact that $q^{*}=q$ and the assumption on $g$, we see that

$$
\begin{aligned}
F^{*}(y, x) & =\sup _{(u, v) \in \mathbb{R}^{2}} u y+v x-q\left(\frac{u+v}{\sqrt{2}}\right)-g\left(\frac{u-v}{\sqrt{2}}\right) \\
& =\sup _{(u, v) \in \mathbb{R}^{2}} \frac{u+v}{2}(x+y)-\frac{u-v}{2}(x-y)-q\left(\frac{u+v}{\sqrt{2}}\right)-g\left(\frac{u-v}{\sqrt{2}}\right) \\
& =\sup _{(u, v) \in \mathbb{R}^{2}} \frac{u+v}{\sqrt{2}} \frac{x+y}{\sqrt{2}}-\frac{u-v}{\sqrt{2}} \frac{x-y}{\sqrt{2}}-q\left(\frac{u+v}{\sqrt{2}}\right)-g\left(\frac{u-v}{\sqrt{2}}\right) \\
& =q^{*}\left(\frac{x+y}{\sqrt{2}}\right)+g^{*}\left(-\frac{x-y}{\sqrt{2}}\right) \\
& =q\left(\frac{x+y}{\sqrt{2}}\right)+g\left(\frac{x-y}{\sqrt{2}}\right) \\
& =F(x, y) .
\end{aligned}
$$

Hence $F$ is autoconjugate. In view of (39), we have $(x, y) \in \operatorname{gra}(G(F)) \Leftrightarrow y \in G(F) x \Leftrightarrow F(x, y)=$ $x y \Leftrightarrow q((x+y) / \sqrt{2})+g((x-y) / \sqrt{2})=x y \Leftrightarrow \frac{1}{4}(x+y)^{2}+g((x-y) / \sqrt{2})=x y \Leftrightarrow \frac{1}{4}(x-y)^{2}+$ $g((x-y) / \sqrt{2})=0 \Leftrightarrow x-y=0 \Leftrightarrow(x, y) \in \operatorname{gra}(\mathrm{Id})$.

Remark 4.3 Consider Theorem 4.2, If we set $g=q=\frac{1}{2}|\cdot|^{2}$, then $F=q \oplus q=q_{\mathrm{Id}} \oplus q_{\mathrm{Id}}^{*}=\mathcal{C}_{\mathrm{Id}}$ by Corollary 2.6. Thus, this pleasingly symmetric choice of $g$ gives rise to $\mathcal{A}_{\text {Id }}=\mathcal{B}_{\text {Id }}=\mathcal{C}_{\text {Id }}$. Proposition 4.1 provides other choices of $g$ that lead to different autoconjugate representers for Id.

Having settled the nonuniqueness of autoconjugate representers, it is natural to ask "What makes the autoconjugate representers of Theorem 3.1 special?" The next result provides a complete answer for a large class of linear operators. 
Theorem 4.4 Let $A: X \rightarrow X^{*}$ be continuous, linear, monotone, and symmetric. Let $F: X \times X^{*} \rightarrow$ ]$-\infty,+\infty]$ be such that $\operatorname{ran} A$ is closed. Then

$$
F=\mathcal{C}_{A} \Leftrightarrow\left\{\begin{array}{l}
F \text { is autoconjugate } \\
F(0,0)=0, \\
(\forall(x, y) \in X \times X) \quad F(x, A y)=F(y, A x) .
\end{array}\right.
$$

Proof. " $\Rightarrow$ ": By Corollary 2.6, $F$ is autoconjugate and $F(0,0)=\left(q_{A} \oplus q_{A}^{*}\right)(0,0)=0$. Let $x$ and $y$ be in $X$. Using (19), we have $F(x, A y)=\left(q_{A} \oplus q_{A}^{*}\right)(x, A y)=q_{A}(x)+q_{A}^{*}(A y)=q_{A}(x)+q_{A}(y)=$ $q_{A}(y)+q_{A}^{*}(A x)=\left(q_{A} \oplus q_{A}^{*}\right)(y, A x)=F(y, A x)$.

" $\Leftarrow$ ": Let $\left(x, x^{*}\right) \in X \times X^{*}$. We proceed by verifying the next two claims.

Claim 1: $x^{*} \notin \operatorname{ran} A \Rightarrow F\left(x, x^{*}\right)=+\infty$.

Assume that $x^{*} \notin \operatorname{ran} A$. The Separation Theorem yields $z \in X$ such that

$$
\left\langle z, x^{*}\right\rangle>0
$$

and $\max \langle z, \operatorname{ran} A\rangle=0$. Since $A$ is symmetric, we deduce that $A z=0$. This implies $(\forall \rho \in \mathbb{R})$ $F(\rho z, 0)=F(\rho z, A 0)=F(0, A(\rho z))=F(0,0)=0$. Thus

$$
\begin{aligned}
(\forall \rho \in \mathbb{R}) \quad F\left(x, x^{*}\right) & =F\left(x, x^{*}\right)+F(\rho z, 0)=F\left(x, x^{*}\right)+F^{*}(0, \rho z) \\
& \geq\langle x, 0\rangle+\left\langle\rho z, x^{*}\right\rangle=\rho\left\langle z, x^{*}\right\rangle .
\end{aligned}
$$

In view of (43), we see that Claim 1 follows by letting $\rho \rightarrow+\infty$ in (44).

Claim 2: $x^{*} \in \operatorname{ran} A \Rightarrow F\left(x, x^{*}\right) \geq \mathcal{C}_{A}\left(x, x^{*}\right)$.

Assume that $x^{*} \in \operatorname{ran} A$, say $x^{*}=A y$. Then $2 F\left(x, x^{*}\right)=2 F(x, A y)=F(x, A y)+F(y, A x)=$ $F(x, A y)+F^{*}(A x, y) \geq\langle x, A x\rangle+\langle y, A y\rangle$ and hence, using (19),

$$
F\left(x, x^{*}\right) \geq q_{A}(x)+q_{A}(y)=q_{A}(x)+q_{A}^{*}(A y)=\left(q_{A} \oplus q_{A}^{*}\right)\left(x, x^{*}\right) .
$$

This and (28) yield Claim 2.

Note that Claim 1 and Claim 2 yield $F \geq \mathcal{C}_{A}$. Therefore, Fact 1.5][iv)] implies that $F=\mathcal{C}_{A}$.

\section{$5 \quad$ Autoconjugate Representers for $\partial(-\ln )$}

Theorem 3.1 showed that three ostensibly different autoconjugate representers are in fact identical for continuous linear monotone operators. It is tempting to consider a subdifferential operator $\partial f$, and to compare $\mathcal{A}_{\partial f}, \mathcal{B}_{\partial f}$, and $f \oplus f^{*}$. It turns out that these autoconjugate representers for $\partial f$ may all be different. To aid in the construction of this example, it will be convenient to work in this section with the negative natural logarithm function

$$
f: \mathbb{R} \rightarrow]-\infty,+\infty]: x \mapsto \begin{cases}-\ln (x), & \text { if } x>0 \\ +\infty, & \text { if } x \leq 0\end{cases}
$$


and with the set

$$
C=\left\{\left(x, x^{*}\right) \in \mathbb{R} \times \mathbb{R} \mid x^{*} \leq-\frac{1}{x}<0\right\}
$$

It is well known that

$$
(\forall x \in \mathbb{R}) \quad f^{*}(x)=-1+f(-x)
$$

and straight-forward to verify that

$$
\begin{gathered}
\frac{1}{\sqrt{2}} C=\left\{\left(x, x^{*}\right) \in \mathbb{R} \times \mathbb{R} \mid x^{*} \leq-\frac{1}{2 x}<0\right\}, \\
\frac{1}{2} C=\left\{\left(x, x^{*}\right) \in \mathbb{R} \times \mathbb{R} \mid x^{*} \leq-\frac{1}{4 x}<0\right\},
\end{gathered}
$$

and

$$
\left.\frac{1}{\sqrt{2}} C \subsetneq \frac{1}{2} C \subsetneq\right] 0,+\infty[\times]-\infty, 0[.
$$

Furthermore, [8, Example 3.4] yields

$$
\left(\forall\left(x, x^{*}\right) \in \mathbb{R} \times \mathbb{R}\right) \quad F_{\partial f}\left(x, x^{*}\right)= \begin{cases}1-2 \sqrt{-x x^{*}}, & \text { if } x \geq 0 \text { and } x^{*} \leq 0 \\ +\infty, & \text { otherwise }\end{cases}
$$

and

$$
F_{\partial f}^{* \top}=-1+\iota_{C}
$$

Theorem 5.1 The functions $\mathcal{A}_{\partial f}, \mathcal{B}_{\partial f}$, and $f \oplus f^{*}$ have domains $\frac{1}{\sqrt{2}} C, \frac{1}{2} C$, and $] 0,+\infty[\times]-\infty, 0[$, respectively. Consequently, they are three different autoconjugate representers for $\partial f$.

Proof. Using (8), (52), (53) and (49), we see that

$$
\begin{aligned}
\operatorname{dom} \mathcal{A}_{\partial f} & =\left\{\left(x, \frac{1}{2} x_{1}^{*}+\frac{1}{2} x_{2}^{*}\right) \in \mathbb{R} \times \mathbb{R} \mid\left(x, x_{1}^{*}\right) \in \operatorname{dom} F_{\partial f} \text { and }\left(x, x_{2}^{*}\right) \in \operatorname{dom} F_{\partial f}^{* \top}\right\} \\
& =\left\{\left(x, \frac{1}{2} x_{1}^{*}+\frac{1}{2} x_{2}^{*}\right) \in \mathbb{R} \times \mathbb{R} \mid x \geq 0, x_{1}^{*} \leq 0, \text { and }\left(x, x_{2}^{*}\right) \in C\right\} \\
& =\left\{\left(x, x^{*}\right) \in \mathbb{R} \times \mathbb{R} \mid x^{*} \leq-\frac{1}{2 x}<0\right\} \\
& =\frac{1}{\sqrt{2}} C,
\end{aligned}
$$

as claimed. Similarly, by (91), (152), and (53),

$$
\begin{aligned}
\operatorname{dom} \mathcal{B}_{\partial f} & =\frac{1}{2} \operatorname{dom} F_{\partial f}+\frac{1}{2} \operatorname{dom} F_{\partial f}^{* \top} \\
& =\frac{1}{2}([0,+\infty[\times]-\infty, 0])+\frac{1}{2} C \\
& =\frac{1}{2} C .
\end{aligned}
$$

Furthermore, by (46) and (48),

$$
\left.\operatorname{dom}\left(f \oplus f^{*}\right)=(\operatorname{dom} f) \times\left(\operatorname{dom} f^{*}\right)=\right] 0,+\infty[\times]-\infty, 0[.
$$

We thus have verified the statements concerning the domains. Fact 1.6, Fact 1.7, and Example 2.5] imply that all three functions are autoconjugate representers for $\partial f$. In view of (51), these functions are all different since their domains are also all different. 
Remark 5.2 Using (52) and (53), one may verify that

$$
\left(\forall\left(x, x^{*}\right) \in \mathbb{R} \times \mathbb{R}\right) \quad \mathcal{A}_{\partial f}\left(x, x^{*}\right)= \begin{cases}-\sqrt{-1-2 x x^{*}}, & \text { if }\left(x, x^{*}\right) \in \frac{1}{\sqrt{2}} C ; \\ +\infty, & \text { otherwise. }\end{cases}
$$

However, we do not have an explicit formula for $\mathcal{B}_{\partial f}$.

\section{Discontinuous Symmetric Operators}

In this final section, we investigate discontinuous symmetric operators. Specifically, we assume throughout this section that $A: X \rightrightarrows X^{*}$ is maximal monotone, at most single-valued, $\operatorname{dom} A$ is a linear subspace, and $\left.A\right|_{\operatorname{dom} A}$ is linear and symmetric. Put differently, we assume that

$A: \operatorname{dom} A \rightarrow X^{*} \quad$ is linear, symmetric, and maximal montone.

It is convenient to extend the definition of $q_{A}$ in (16) to this more general setting via

$$
q_{A}: X \rightarrow \mathbb{R}: x \mapsto \begin{cases}\frac{1}{2}\langle x, A x\rangle, & \text { if } x \in \operatorname{dom} A \\ +\infty, & \text { otherwise. }\end{cases}
$$

A key tool is the function

$$
f: X \rightarrow]-\infty,+\infty]: x \mapsto \sup _{y \in \operatorname{dom} A}\langle x, A y\rangle-\frac{1}{2}\langle y, A y\rangle
$$

which was introduced by Phelps and Simons.

Fact 6.1 (Phelps-Simons) (See [32].) The following hold.

(i) $f$ is proper, lower semicontinuous, and convex.

(ii) $A=\partial f$.

(iii) $\operatorname{dom} A \subseteq \operatorname{dom} f \subseteq \overline{\operatorname{dom}} A$ and $(\forall x \in \operatorname{dom} A) f(x)=\frac{1}{2}\langle x, A x\rangle$.

(iv) $A$ is continuous $\Leftrightarrow \operatorname{dom} A=X \Leftrightarrow \operatorname{dom} f=X$.

Corollary 6.2 The following hold.

(i) $f+\iota_{\operatorname{dom} A}=q_{A}$.

(ii) $f=q_{A} \Leftrightarrow \operatorname{dom} f=\operatorname{dom} A$.

(iii) $q_{A}^{* *}=f$.

(iv) If $A$ is one-to-one, then $f=q_{A^{-1}}^{*}$. 
Proof. (i); Clear from Fact 6.1](iii), (ii); Since $\operatorname{dom} q_{A}=\operatorname{dom} A$, this item is a consequence of (i), (iii): Using Fact 6.1)(i) \& (ii) and a result by J. Borwein (see [11, Theorem 1] or [45, Theorem 3.1.4(i)]), we see that $f=f^{* *}=\left(f+\iota_{\operatorname{dom} \partial f}\right)^{* *}=\left(f+\iota_{\operatorname{dom} A}\right)^{* *}=q_{A}^{* *} \cdot$ (iv); If $A$ is one-to-one, then

$$
(\forall x \in X) \quad f(x)=\sup _{y^{*} \in \operatorname{dom} A^{-1}}\left\langle x, y^{*}\right\rangle-\frac{1}{2}\left\langle A^{-1} y^{*}, y^{*}\right\rangle=\sup _{y^{*} \in \operatorname{dom} q_{A^{-1}}}\left\langle x, y^{*}\right\rangle-q_{A^{-1}}\left(y^{*}\right)=q_{A^{-1}}^{*}(x) .
$$

This completes the proof.

Proposition 6.3 We have: $\operatorname{dom} A=\operatorname{dom} f \Leftrightarrow$ every sequence $\left(x_{n}\right)_{n \in \mathbb{N}}$ in $\operatorname{dom} A$ such that $\left(x_{n}\right)_{n \in \mathbb{N}}$ and $\left(\left\langle x_{n}, A x_{n}\right\rangle\right)_{n \in \mathbb{N}}$ are convergent must satisfy $\lim x_{n} \in \operatorname{dom} A$.

Proof. " $\Rightarrow$ ": Assume that $\left(x_{n}\right)_{n \in \mathbb{N}}$ is a sequence in $\operatorname{dom} A$ such that $\left(x_{n}\right)_{n \in \mathbb{N}}$ converges to $x \in X$ and $\left(\left\langle x_{n}, A x_{n}\right\rangle\right)_{n \in \mathbb{N}}$ is also convergent. Using Fact 6.1) and Corollary 6.2)(i), we have $x \in \operatorname{dom} f$ and thus $x \in \operatorname{dom} A$.

" $\Leftarrow$ ": In view of Fact 6.1](iii), it suffices to show that $\operatorname{dom} f \subseteq \operatorname{dom} A$. To this end, let $x \in \operatorname{dom} f$. In view of Corollary 6.2(iii), there exists a sequence $\left(x_{n}\right)_{n \in \mathbb{N}}$ in $\operatorname{dom} q_{A}=\operatorname{dom} A$ such $x_{n} \rightarrow x$ and $\frac{1}{2}\left\langle x_{n}, A x_{n}\right\rangle=q_{A}\left(x_{n}\right) \rightarrow f(x)$. By assumption, $x \in \operatorname{dom} A$, as required.

Theorem 6.4 Let $B: X^{*} \rightarrow X$ be continuous, linear, symmetric, monotone, and one-to-one. Suppose that $A=B^{-1}$. Then

$$
\mathcal{A}_{A}=q_{A} \oplus q_{B}=\left(q_{B}^{*}+\iota_{\operatorname{dom} A}\right) \oplus q_{B}
$$

and

$$
\mathcal{B}_{A}=\mathcal{A}_{A}^{* *}=q_{B}^{*} \oplus q_{B}
$$

are both representers for $A$. Furthermore, $\mathcal{A}_{A}=\mathcal{B}_{A} \Leftrightarrow \operatorname{dom} q_{B}^{*}=\operatorname{dom} A$.

Proof. Since $q_{A}=q_{B}^{*}+\iota_{\mathrm{dom} A}$ by Corollary 6.2)(i) $\&$ (iv), it suffices to verify the left equality in (62). Let $\left(x, x^{*}\right) \in X \times X^{*}$. Using (8) and Fact 1.2](ii), we see that

$$
\begin{aligned}
\mathcal{A}_{A}\left(x, x^{*}\right) & =\inf _{y^{*}} \frac{1}{2} F_{A}\left(x, x^{*}+y^{*}\right)+\frac{1}{2} F_{A}^{*}\left(x^{*}-y^{*}, x\right) \\
& =\inf _{y^{*}} \frac{1}{2} F_{B}\left(x^{*}+y^{*}, x\right)+\frac{1}{2} F_{B}^{*}\left(x, x^{*}-y^{*}\right) \\
& =\inf _{y^{*}} \frac{1}{2} F_{B}\left(x^{*}+y^{*}, x\right)+\frac{1}{2}\left(\iota_{\operatorname{gra} B}\left(x^{*}-y^{*}, x\right)+\left\langle x^{*}-y^{*}, B\left(x^{*}-y^{*}\right)\right\rangle\right) .
\end{aligned}
$$

If $x \notin \operatorname{ran} B=\operatorname{dom} A$, then (64) shows that $\mathcal{A}_{A}\left(x, x^{*}\right)=+\infty$, as required. So assume that $x \in \operatorname{ran} B=\operatorname{dom} A$. In view of (64), (21), and (19), we deduce that

$$
\begin{aligned}
\mathcal{A}_{A}\left(x, x^{*}\right) & =\frac{1}{2} F_{B}\left(2 x^{*}-A x, x\right)+\frac{1}{2}\langle x, A x\rangle \\
& =q_{B}^{*}\left(\frac{1}{2} x+\frac{1}{2} B\left(2 x^{*}-A x\right)\right)+q_{A}(x) \\
& =q_{B}^{*}\left(B x^{*}\right)+q_{A}(x) \\
& =q_{B}\left(x^{*}\right)+q_{A}(x) .
\end{aligned}
$$


Hence (62) holds. Using (62), Corollary 6.2)(iii) \& (iv), (28), and Theorem [3.1, we see that

$$
\mathcal{A}_{A}^{* *}=\left(q_{A} \oplus q_{B}\right)^{* *}=q_{A}^{* *} \oplus q_{B}^{* *}=q_{B}^{*} \oplus q_{B}=\left(q_{B} \oplus q_{B}^{*}\right)^{*}=\mathcal{B}_{B}^{*}=\mathcal{B}_{B}^{\top}=\mathcal{B}_{B^{-1}}=\mathcal{B}_{A},
$$

so that (63) holds. Furthermore, $\mathcal{A}_{A}=\mathcal{B}_{A} \Leftrightarrow q_{A} \oplus q_{B}=q_{A}^{* *} \oplus q_{B} \Leftrightarrow q_{A}=q_{A}^{* *} \Leftrightarrow \operatorname{dom} q_{A}^{* *}=\operatorname{dom} A$ $\Leftrightarrow \operatorname{dom} q_{B}^{*}=\operatorname{dom} A$ by Corollary 6.2 .

Example 6.5 Suppose that $X$ is the Hilbert space $\ell_{2}(\mathbb{N})$ of square-summable sequences; thus, $X^{*}=X$. Set

$$
B: X \rightarrow X:\left(\xi_{k}\right)_{k \in \mathbb{N}} \mapsto\left(\frac{1}{k} \xi_{k}\right)_{k \in \mathbb{N}}
$$

and suppose that $A=B^{-1}$. Then $\operatorname{ran} B=\operatorname{dom} A$ is dense in $X$, but it is not closed (since, e.g., $\left.\left(\frac{1}{k}\right)_{k \in \mathbb{N}} \in X \backslash(\operatorname{ran} B)\right)$. Now set

$$
x=\left(\frac{1}{k^{4 / 3}}\right)_{k \in \mathbb{N}} \text { and } \quad(\forall n \in \mathbb{N}) x_{n}=\left(\frac{1}{1^{4 / 3}}, \frac{1}{2^{4 / 3}}, \ldots, \frac{1}{n^{4 / 3}}, 0,0, \ldots\right) .
$$

On the one hand, $\left(x_{n}\right)_{n \in \mathbb{N}}$ lies in $\operatorname{dom} A$ and $x_{n} \rightarrow x \in X \backslash(\operatorname{dom} A)$. On the other hand, $\left\langle x_{n}, A x_{n}\right\rangle=\sum_{k=1}^{n} \frac{1}{k^{4 / 3}} \frac{k}{k^{4 / 3}}=\sum_{k=1}^{n} \frac{1}{k^{5 / 3}} \rightarrow \zeta(5 / 3) \in \mathbb{R}$. Altogether, Proposition 6.3 implies that $\operatorname{dom} A \subsetneq \operatorname{dom} q_{B}^{*}$. Therefore, by Theorem 6.4, $\mathcal{A}_{A}$ is neither lower semicontinuous nor equal to $\mathcal{B}_{A}$. While $\mathcal{A}_{A}$ is still a representer for $A$, it cannot be autoconjugate.

Remark 6.6 Several comments are in order.

(i) Without the constraint qualification, Fact 1.6 fails (see Example 6.5, where $\operatorname{dom} A$ is a subspace that is not closed).

(ii) It is conceivable that $\mathcal{A}_{A}^{* *}$ is always an autoconjugate representer for $A$ - this would sharpen Fact 1.6 and it would be consistent with Theorem 6.4.

(iii) Suppose that $B$ is as in Theorem 6.4, that $A=B^{-1}$, and that $\operatorname{dom} A=\operatorname{ran} B$ is a dense subspace of $X$ with $\operatorname{dom} A \neq X$.

We do not know whether $(\operatorname{dom} f) \backslash(\operatorname{dom} A) \neq \varnothing$ must hold (as it does in Example 6.5), i.e. (see Proposition [6.3), whether there exists a sequence $\left(x_{n}\right)_{n \in \mathbb{N}}$ in $\operatorname{dom} A$ such that $\left(x_{n}\right)_{n \in \mathbb{N}}$ converges to some point $x \in X \backslash(\operatorname{dom} A)$, yet $\left(\left\langle x_{n}, A x_{n}\right\rangle\right)_{n \in \mathbb{N}}$ converges to a real number.

In contrast, there does exist a point $x \in X \backslash(\operatorname{dom} A)$ such that every sequence $\left(x_{n}\right)_{n \in \mathbb{N}}$ in $\operatorname{dom} A$ converging to $x$ must have $\left\langle x_{n}, A x_{n}\right\rangle \rightarrow+\infty$. (Indeed, since $\operatorname{dom} A \neq X$, it follows from Fact 6.1](iv) that $\operatorname{dom} f \neq X$. Take $x \in X \backslash(\operatorname{dom} f)$ and assume that $\left(x_{n}\right)_{n \in \mathbb{N}}$ lies in $\operatorname{dom} A$ and converges to $x$. Then $+\infty=f(x) \leq \underline{\lim } f\left(x_{n}\right)=\underline{\lim } \frac{1}{2}\left\langle x_{n}, A x_{n}\right\rangle$ by Fact 6.1](i) \& (iii)]. Thus for every sequence $\left(x_{n}^{*}\right)_{n \in \mathbb{N}}$ in $X^{*}$ such that $B x_{n}^{*} \rightarrow x \notin \operatorname{ran} B$, it follows that $\left\|B x_{n}^{*}\right\| \cdot\left\|x_{n}^{*}\right\|_{*} \geq\left\langle B x_{n}^{*}, x_{n}^{*}\right\rangle=\left\langle B x_{n}^{*}, A\left(B x_{n}^{*}\right)\right\rangle \rightarrow+\infty$. Since $0 \in \operatorname{dom} f$ and so $x \neq 0$, we deduce $\left\|x_{n}^{*}\right\|_{*} \rightarrow+\infty$, which is a well known result from Functional Analysis (see [25, Corollary 17.G]). 


\section{Acknowledgment}

Heinz Bauschke was partially supported by the Canada Research Chair Program and by the Natural Sciences and Engineering Research Council of Canada. Xianfu Wang was partially supported by the Natural Sciences and Engineering Research Council of Canada.

\section{References}

[1] S. Bartz, H. H. Bauschke, J. M. Borwein, S. Reich, and X. Wang, "Fitzpatrick functions, cyclic monotonicity, and Rockafellar's antiderivative", Nonlinear Analysis, vol. 66, pp. 1198-1223, 2007.

[2] H. H. Bauschke, "Fenchel duality, Fitzpatrick functions and the extension of firmly nonexpansive mappings", Proceedings of the American Mathematical Society, vol. 135, pp. 135-139, 2007.

[3] H. H. Bauschke, J. M. Borwein, and X. Wang, "Fitzpatrick functions and continuous linear monotone operators", SIAM Journal on Optimization, vol. 18, pp. 789-809, 2007.

[4] H. H. Bauschke, R. Goebel, Y. Lucet, and X. Wang, "The proximal average: basic theory", submitted.

[5] H. H. Bauschke, Y. Lucet, and M. Trienis, "How to transform one convex function continuously into another", SIAM Review, vol. 50, pp. 115-132, 2008.

[6] H. H. Bauschke, Y. Lucet, and X. Wang, "Primal-dual symmetric antiderivatives for cyclically monotone operators", SIAM Journal on Control and Optimization, vol. 46, pp. 2031-2051, 2007.

[7] H. H. Bauschke, E. Matoušková, and S. Reich, "Projection and proximal point methods: convergence results and counterexamples", Nonlinear Analysis, vol. 56, pp. 715-739, 2004.

[8] H. H. Bauschke, D. A. McLaren, and H. S. Sendov, "Fitzpatrick functions: inequalities, examples and remarks on a problem by S. Fitzpatrick", Journal of Convex Analysis, vol. 13, pp. 499-523, 2006.

[9] H. H. Bauschke and X. Wang, "The kernel average for two convex functions and its applications to the extension and representation of monotone operators", Transactions of the American Mathematical Society, to appear.

[10] H. H. Bauschke and X. Wang, "A convex-analytical approach to extension results for $n$ cyclically monotone operators", Set-Valued Analysis, vol. 15, pp. 297-306, 2007.

[11] J. M. Borwein, "A note on $\varepsilon$-subgradients and maximal monotonicity", Pacific Journal of Mathematics, vol. 103, pp. 307-314, 1982. 
[12] J. M. Borwein, "Maximal monotonicity via convex analysis", Journal of Convex Analysis, vol. 13, pp. 561-586, 2006.

[13] J. M. Borwein, "Maximality of sums of two maximal monotone operators", Proceedings of the American Mathematical Society, vol. 134, pp. 2951-2955, 2006.

[14] J. M. Borwein and Q. J. Zhu, Techniques of Variational Analysis, Springer-Verlag, 2005.

[15] R. I. Boţ, E. R. Csetnek, and G. Wanka, "A new condition for maximal monotonicity via representative functions", Nonlinear Analysis, vol. 67, pp. 2390-2402, 2007.

[16] R. I. Bot,, S.-M. Grad, and G. Wanka, "Weaker constraint qualifications in maximal monotonicity", Numerical Functional Analysis and Optimization, vol. 28, pp. 27-41, 2007.

[17] R. S. Burachik and S. Fitzpatrick, "On the Fitzpatrick family associated to some subdifferentials", Journal of Nonlinear and Convex Analysis, vol. 6, pp. 165-171, 2005.

[18] R. S. Burachik and A. N. Iusem, "On non-enlargeable and fully enlargeable monotone operators", Journal of Convex Analysis, vol. 13, pp. 603-622, 2006.

[19] R. S. Burachik and A. N. Iusem, Set-Valued Mappings and Enlargements of Monotone Operators, Springer-Verlag, 2007.

[20] R. S. Burachik and B. F. Svaiter, "Operating enlargements of monotone operators: new connections with convex functions", Pacific Journal of Optimization, vol. 2, pp. 425-445, 2006.

[21] S. Fitzpatrick, "Representing monotone operators by convex functions," Workshop/Miniconference on Functional Analysis and Optimization (Canberra 1988), Proceedings of the Centre for Mathematical Analysis, Australian National University vol. 20, Canberra, Australia, pp. 59-65, 1988.

[22] Y. García, M. Lassonde, and J. Revalski, "Extended sums and extended compositions of monotone operators", Journal of Convex Analysis, vol. 13, pp. 721-738, 2006.

[23] N. Ghoussoub, "Maximal monotone operators are selfdual vector fields and vice-versa", 2006, preprint available at http://aps.arxiv.org/abs/math/0610494v1.

[24] J.-B. Hiriart-Urruty and C. Lemaréchal, Convex Analysis and Minimization Algorithms II, Springer-Verlag, 1996.

[25] R. B. Holmes, Geometric Functional Analysis and its Applications, Springer-Verlag, 1975.

[26] J.-E. Martínez-Legaz and C. F. Svaiter, "Monotone operators representable by l.s.c. convex functions", Set-Valued Analysis, vol. 13, pp. 21-46, 2005.

[27] J.-E. Martínez-Legaz and M. Théra, "A convex representation of maximal monotone operators", Journal of Nonlinear and Convex Analysis, vol. 2, pp. 243-247, 2001.

[28] J.-P. Penot, "Autoconjugate functions and representations of monotone operators", Bulletin of the Australian Mathematics Soceity, vol. 67, pp. 277-284, 2003. 
[29] J.-P. Penot, "The relevance of convex analysis for the study of monotonicity", Nonlinear Analysis, vol. 58, pp. 855-871, 2004.

[30] J.-P. Penot and C. Zălinescu, "Some problems about the representation of monotone operators by convex functions", The Australian New Zealand Industrial and Applied Mathematics Journal, vol. 47, pp. 1-20, 2005.

[31] R. R. Phelps, Convex Functions, Monotone Operators and Differentiability, 2nd Edition, Springer-Verlag, 1993.

[32] R. R. Phelps and S. Simons, "Unbounded linear monotone operators on nonreflexive Banach spaces", Journal of Convex Analysis, vol. 5, pp. 303-328, 1998.

[33] S. Reich and S. Simons, "Fenchel duality, Fitzpatrick functions and the Kirszbraun-Valentine extension theorem", Proceedings of the American Mathematical Society, vol. 133, pp. 2657$2660,2005$.

[34] R. T. Rockafellar, Convex Analysis, Princeton University Press, 1970.

[35] R. T. Rockafellar and R. J-B Wets, Variational Analysis, Springer-Verlag, 1998.

[36] S. Simons, Minimax and Monotonicity, Springer-Verlag, 1998.

[37] S. Simons, "Dualized and scaled Fitzpatrick functions", Proceedings of the American Mathematical Society, vol. 134, pp. 2983-2987, 2006.

[38] S. Simons, "LC-functions and maximal monotonicity", Journal of Nonlinear and Convex Analyis, vol. 7, pp. 123-138, 2006.

[39] S. Simons, "The Fitzpatrick function and nonreflexive spaces", Journal of Convex Analysis, vol. 13, pp. 861-881, 2006.

[40] S. Simons, From Hahn-Banach to Monotonicity, Springer-Verlag, to appear.

[41] S. Simons and C. Zălinescu, "A new proof for Rockafellar's characterization of maximal monotone operators", Proceedings of the American Mathematical Society, vol. 132, pp. 2969-2972, 2004.

[42] S. Simons and C. Zălinescu, "Fenchel duality, Fitzpatrick functions and maximal monotonicity", Journal of Nonlinear and Convex Analysis, vol. 6, pp. 1-22, 2005.

[43] B. F. Svaiter "Fixed points in the family of convex representations of a maximal monotone operator", Proceeding of the American Mathematical Society, vol. 131, pp. 3851-3859, 2003.

[44] M. D. Voisei, "A maximality theorem for the sum of maximal monotone operators in nonreflexive Banach spaces", Mathematical Sciences Research Journal, vol. 10, pp. 36-41, 2006.

[45] C. Zălinescu, Convex Analysis in General Vector Spaces, World Scientific Publishing, 2002.

[46] C. Zălinescu, "A new proof of the maximal monotonicity of the sum using the Fitzpatrick function", in Variational Analysis and Applications, pp. 1159-1172, Springer-Verlag, 2005. 